Version of Record: https://www.sciencedirect.com/science/article/pii/S1359645419300333

Manuscript_0de567768963c3f710bf866f2bb49329

\title{
Experimental study of asymmetrical tilt boundaries in WC-Co alloys
}

Maxime Pellan ${ }^{*}$, Sabine Lay ${ }^{1}$, Jean-Michel Missiaen

Univ. Grenoble Alpes, CNRS, Grenoble INP, SIMAP, 38000 Grenoble, France

*Present address, Sandvik Hyperion SAS, Grenoble, France

\begin{abstract}
.
The rotation angle distribution of grain boundaries with [2 - 1 - -10$]$ misorientation axis in WCCo alloys was investigated by electron backscatter diffraction for a range of cobalt contents. The effect of carbon content and sintering time was also studied. Preferred misorientations correspond to low-index boundary habit planes involving the basal or prismatic plane for at least one grain. The $26.2^{\circ}$ [ $2-1-10$ ] boundary was imaged by high resolution transmission electron microscopy. The joining of the mismatched planes at the boundary is accommodated by dislocations which are interpreted with reference to the $29.4^{\circ}$ [ $2-1-10$ ] boundary. The dislocation spacing and direction found experimentally are in agreement with calculated values obtained by the 02-lattice approach taking into account the misorientation and parametric misfit. The periodicity and characteristics of the dislocations indicate an optimization of the grain boundary structure and energy.
\end{abstract}

\section{Keywords}

Asymmetrical tilt grain boundaries; WC-Co alloys; misfit dislocations; TEM; EBSD.

\section{Introduction}

Among hardmetals, cemented carbides play a critical role in numerous industrial processes such as metal cutting, mining or drilling [1,2]. They are mainly based on WC-Co alloys and consist of hard WC grains in a ductile Co rich matrix. The physical properties of a composite material depend on the size and distribution of its components. While the contiguity of the WC phase is very high in alloys with low cobalt content, it decreases and seems to reach a plateau for high Co contents, independently of the WC grain size [3-5]. The existence of a more or less continuous WC skeleton across the alloy influences its mechanical performance. Better knowledge of the WC grain boundary population in the alloy should ensure a better design of the microstructure of this widely used hard material.

For WC-Co alloys, electron backscatter diffraction (EBSD) provided relevant statistical data on the habit plane and misorientation of WC grain boundaries [6-9]. About $60 \%$ of the boundaries are parallel to a basal or prismatic plane of at least one of the two adjoining crystals. Several special high angle boundaries were identified in the alloys, mainly the socalled $\Sigma=2,13$ and 97 boundaries using the coincidence site lattice description (CSL) and assuming a parametric ratio $\mathrm{c} / \mathrm{a}=1[10]$. In this framework, the grain boundaries correspond

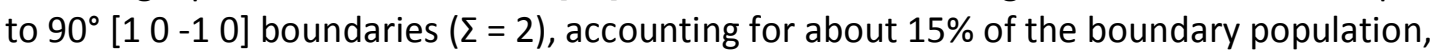
$27.8^{\circ}\left[\begin{array}{llll}0 & 0 & 0 & 1\end{array}\right]$ boundaries $(\Sigma=13)$ representing about 1-3\% and 90 $\left[\begin{array}{lll}2-1 & -1 & 0\end{array}\right](\Sigma=97)$ in

\footnotetext{
${ }^{1}$ Corresponding author : Sabine LAY, sabine.lay@simap.grenoble-inp.fr, 33476826628

SIMAP, Domaine Universitaire

1130, rue de la piscine, BP 75

38402 Saint Martin d'Hères
} 
much smaller amounts. In a previous study, the deviation of the $\Sigma=2$ grain boundary from the ideal $90^{\circ}$ [10-10] misorientation was analyzed [11]. Atomistic calculations performed on WC grain boundaries indicate that the lowest energy is associated with $\Sigma=2$ boundaries $[12,13]$. The carbon content in the alloy also influences the WC/Co interface and WC grain boundary energies [14].

EBSD characterization in WC-Co alloys with a high Co content emphasizes that most grain boundaries are not special boundaries, but little is known about their characteristics. This work is a step towards gaining a better knowledge of stable boundaries in cemented carbides. It also provides new experimental results on grain boundaries with no rational match at the habit plane. Little experimental data on the atomic arrangement in such boundaries are available right now although they probably represent most of the grain boundaries in actual materials.

The study focuses on WC grain boundaries whose misorientation may be described by a rotation about the [2 $-1-10$ ] axis. These grain boundaries, called [2 -1 -1 0] boundaries in what follows, had not attracted much study [15]. In this work, they are investigated both by EBSD and by transmission electron microscopy (TEM). The effect of Co content, which monitors the contiguity of the WC phase, is studied. The influence of sintering time is also explored, as a long heat treatment should lead to a decrease in high energy grain boundaries. Moreover, the effect of carbon potential in the alloy is examined. The grain boundary stability is questioned regarding the misorientation and atomic structure. The effect of low energy boundary habit planes of WC is highlighted.

\section{Experimental procedure}

WC-Co alloys with 10, 15, 20, 30, 40 and 50 vol \% Co were sintered at $1410^{\circ} \mathrm{C}$ for 1 and $5 \mathrm{~h}$ with a WC powder mean grain size of $0.6 \mu \mathrm{m}$. For each Co content, two sets of alloys were prepared with an excess of C or of W, called C2 and W2, respectively (Fig. 1). Their composition lies in the two phase domain at the limit with the three phase domains at $1000^{\circ} \mathrm{C}[16]$. Grain boundaries were studied both in the WC powder and in the sintered alloys. For the WC powder, organic compounds and cobalt were removed from a powder mixture after milling and WC powder was consolidated by sintering with $40 \mathrm{wt} \% \mathrm{Cu}$ at $1120^{\circ} \mathrm{C}$ for 15 min under $\mathrm{He} / \mathrm{H} 2$ atmosphere. Solubility in $\mathrm{Cu}$ is sufficiently low at this temperature to ensure that WC powder is unchanged by the preparation. An additional WC20 vol\% Co alloy called WC-20Co,Cr,C was used in this work because the atomic structure of an asymmetric tilt boundary could be studied in this alloy. The sample containing $0.65 \mathrm{at} \% \mathrm{Cr}$ and an excess of carbon was sintered at $1200^{\circ} \mathrm{C}$ for $1 \mathrm{~h}$, i.e. slightly under the temperature of liquid formation determined as $1220^{\circ} \mathrm{C}$ [17]. 

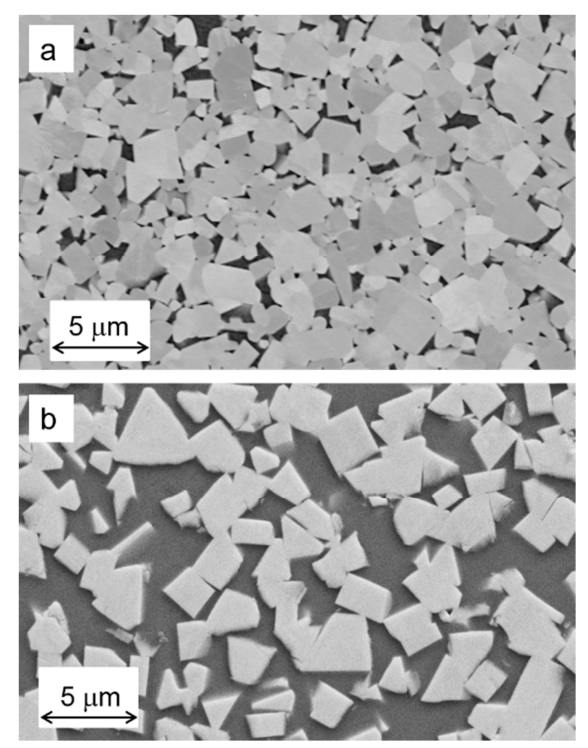

Figure 1. Scanning electron microscopy images of (a) W2-10Co and (b) C2-50Co alloys sintered for $1 \mathrm{~h}$. The WC phase appears as bright grains surrounded by the Co rich binder in the back scattered electron mode.

Sintered samples were mechanically polished down to $1 / 10 \mu \mathrm{m}$, then Ar-milled for $2 \mathrm{~h}$ with a $2^{\circ}$ incident angle and $2 \mathrm{kV}$ accelerating voltage for EBSD characterization. Five $30 \times 30 \mu \mathrm{m}$ maps were collected for each sample with a step size of $40 \mathrm{~nm}$ at an accelerating voltage of $20 \mathrm{kV}$. Grain mapping was obtained using a minimum misorientation for grain boundaries of $2^{\circ}$ and a minimum grain size of 6 pixels $(0.1 \mu \mathrm{m})$. Between 800 and 1200 grains were analysed per sample. Orientation information was extracted from the EBSD maps using the Aphelion $^{\mathrm{TM}}$ image analysis software. WC boundary misorientations were determined by a dedicated Matlab ${ }^{\text {TM }}$ program [8].

Among the twelve equivalent descriptions for boundary misorientations in the hexagonal structure, the angle-axis pairs associated with the smallest angle were chosen. According to experimental conditions, the angular resolution on the misorientation angle at a grain boundary in EBSD is considered to be close to $0.5^{\circ}$ [18]. In this work, a tolerance angle of $5^{\circ}$ on the orientation of the rotation axis was used to analyse the boundary population in the alloys. With these parameters, about 350 grain boundaries were found with [2 -1 -1 0 ] rotation axis in the alloys with the lowest Co content and down to 40 in the alloys with the highest Co content. The whole analysis covers more than 4000 [2-1-10] boundaries in the sintered alloys. Transmission electron microscopy (TEM) observations were conducted using a JEOL 3010 microscope running at $300 \mathrm{kV}$. TEM thin foils were prepared by mechanical grinding and ion beam milling. Complementary high resolution TEM observations (HRTEM) were performed in the WC-20Co,Cr,C alloy using the 4000EX JEOL microscope running at 400 kV.

\section{Results}




\subsection{Frequency of [ $2-1-10]$ boundaries in WC-Co alloys}

The distribution of grain boundary misorientation axes was analysed from EBSD data for comparison between WC powder and sintered alloys. The most frequent axis lies close to [0

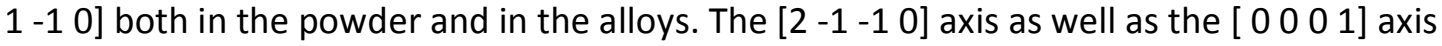
are also visible but in much lower numbers [9].

In order to obtain a precise knowledge on the preponderance of [2 -1 -1 0] boundaries, their relative frequency was determined in all alloys and in the powder (Fig. 2). Sintering increases the number of such boundaries. Their frequency also increases with the cobalt content. However, no effect of carbon potential or sintering time is observed. For high Co contents, the increase in [ $2-1-10]$ boundaries occurs at the expense of random boundaries. This is probably facilitated by the easier rearrangement of the WC skeleton and indicates a higher stability of these boundaries.

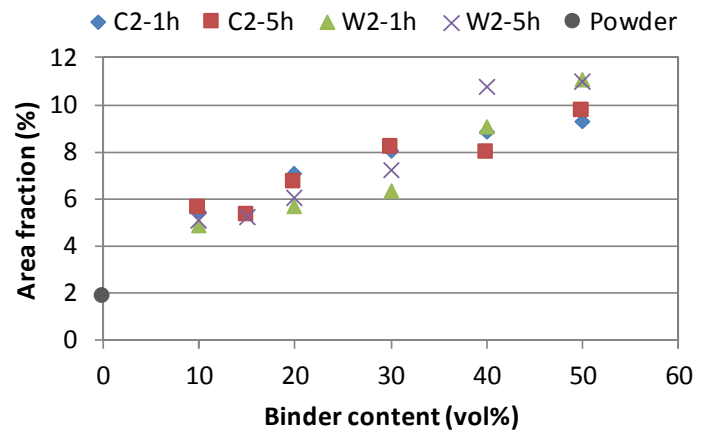

Figure 2. Area fraction of [2 - $1-10$ ] boundaries as a function of Co content and sintering time for the two sets of alloys ( $C 2$ and W2). The area fraction was expressed per unit solid angle.

The rotation angle distribution for the [ $2-1-10]$ axis reveals interesting features (Fig. 3 ). Although the extent of [2-1-1 0] boundaries is small in the powder, a peak is observed for $\theta$ $\approx 60^{\circ}$ (Fig. 3a). The angle distribution was also analysed for all sintered alloys separately to detect an effect of sintering time, Co content or $C$ potential. However, no significant influence of these parameters was found. The results obtained for all alloys are therefore depicted together on the same graph (Fig. 3b). A broad distribution of rotation angles is noticed but several peaks are detected, mainly close to $43^{\circ}, 49^{\circ}, 60^{\circ}$ and $90^{\circ}$. The 60 and $90^{\circ}$ rotation angles correspond to $\Sigma=4$ and 97 boundaries, respectively.

The frequency of the three main [ $2-1-10$ ] boundaries revealed by EBSD measurements in the alloys is shown in figure 4 in surface fraction relative to all boundaries. Note that the values measured for the highest Co contents are less reliable due to the smaller number of WC grains in the investigated areas. The surface fraction of $60^{\circ}$ boundaries close to $1 \%$ in the powder slightly increases to about $1.5 \%$ with Co content. The $49^{\circ}$ and $90^{\circ}$ boundaries mainly form during sintering and reach about 1.5 - $2 \%$ which suggests that these grain boundaries are energetically favoured. 

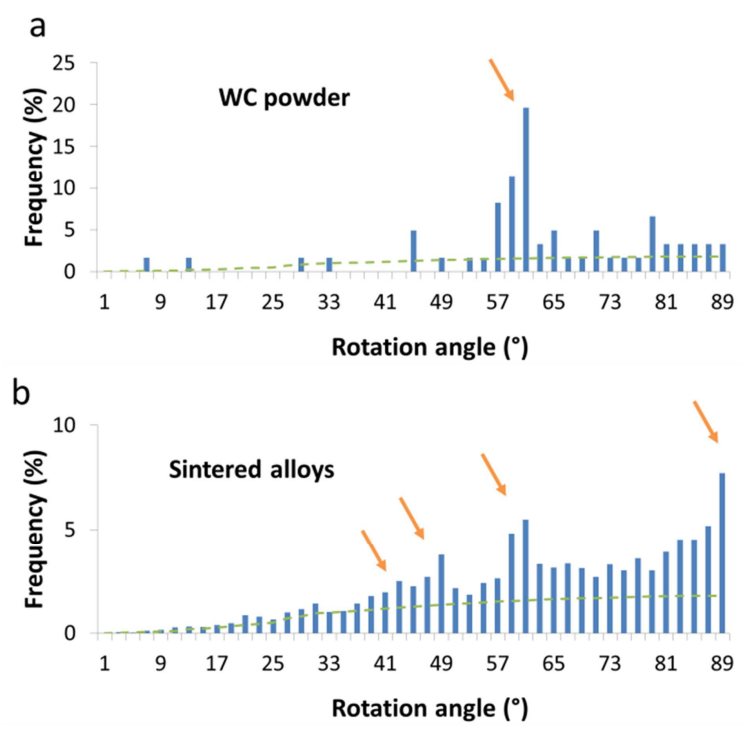

Figure 3. Rotation angle distribution for the [ $2-1-1$ 0] axis (a) for the WC powder and (b) in the sintered alloys. The frequency in number is relative to the [2 -1 -1 0] grain boundaries. The plot of a random distribution in a hexagonal material is drawn as a dotted line [19].
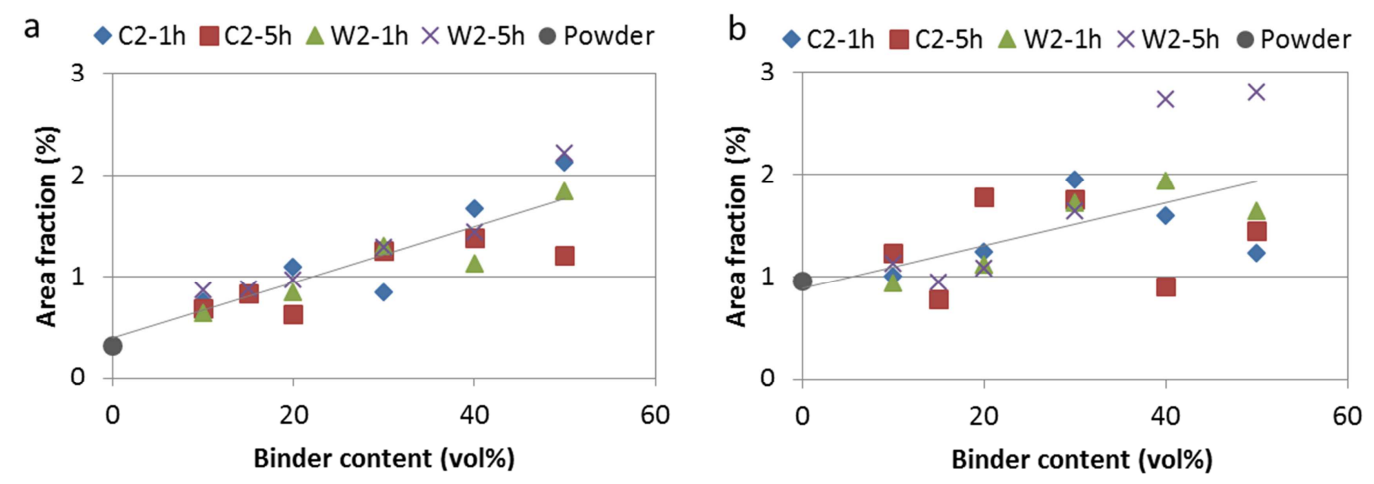

C

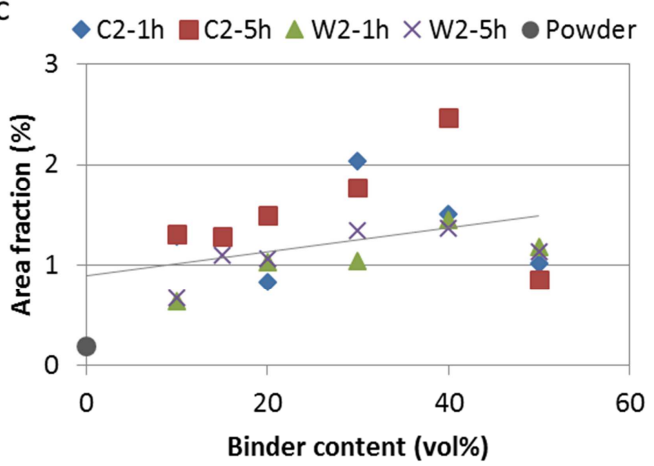

Figure 4. Surface fraction of [ $2-1-10$ ] boundaries compared to all boundaries in the material with rotation angle equal to (a) $49^{\circ}$, (b) $60^{\circ}$ and (c) $90^{\circ}$. The tolerance angle on the rotation angle and axis direction is $5^{\circ}$. 


\subsection{Habit planes of [ $2-1-10]$ grain boundaries}

TEM observations were carried out to obtain more precise data on the habit planes of these boundaries. In sintered alloys, the WC grain shape is close to a prism with a triangular base,

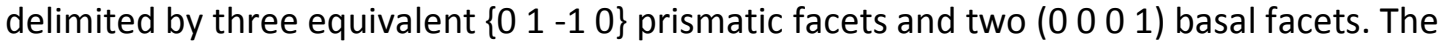
observation direction of WC grains was chosen parallel to [2 -1 -1 0$]$ to allow direct measurement of the rotation angle at the boundary and identification of the boundary plane (Fig. 5).

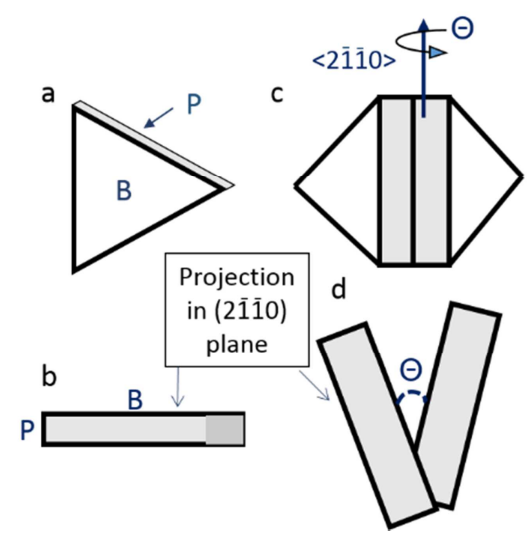

Figure 5. (a) WC grain delimited by two basal facets (B) and three prismatic facets $(P)$ in WCCo alloys. (b) Its projection along [ $2-1-10$ ] with two $B$ and only one $P$ facet parallel to the [2

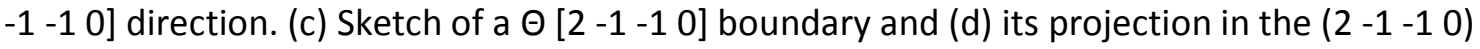
plane.

The striking feature is that the boundaries have an asymmetrical tilt character with the habit plane being a basal or prismatic facet for at least one grain. The other plane is defined by the rotation angle. Figure 6 shows several examples of such asymmetrical tilt boundaries where

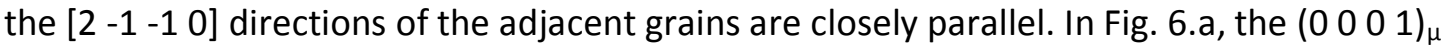
plane is parallel to the $\left(\begin{array}{llll}0 & -1 & 1 & 1\end{array}\right)_{\lambda}$ plane, in Fig. $5 . b$ to the $(0-221){ }_{\lambda}$ plane, in Fig. 5.c, the prismatic plane is parallel to the $(01-12)_{\lambda}$ plane. In these cases, flat boundaries are found.
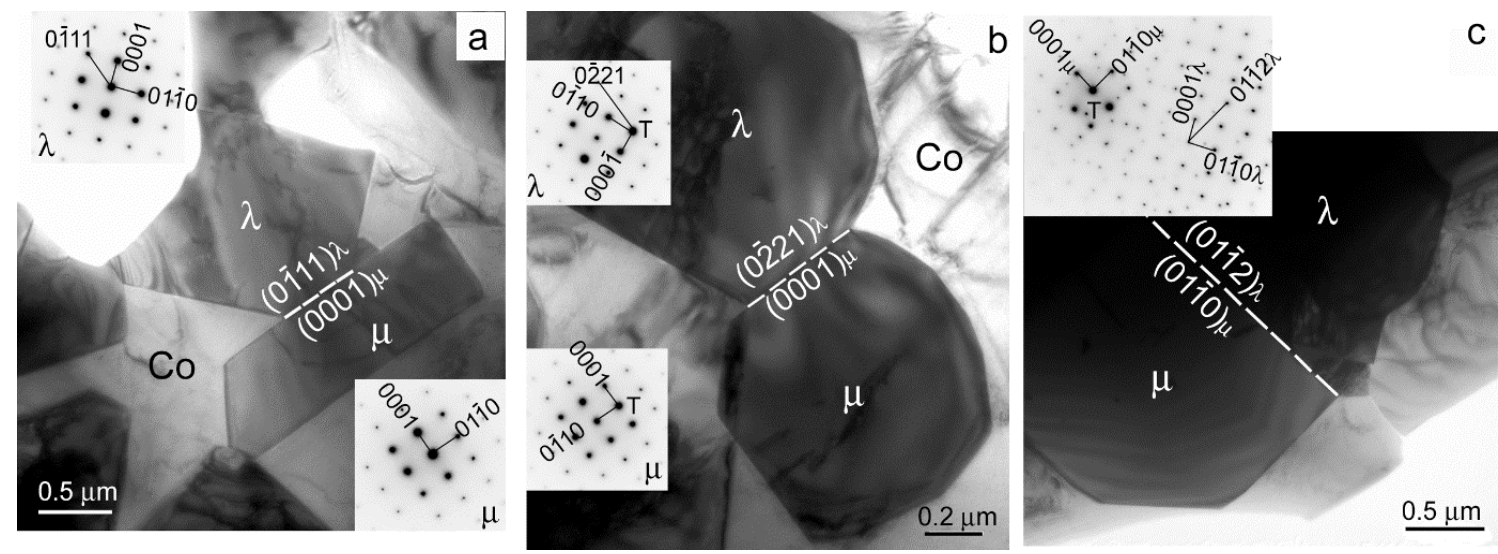
Figure 6: Examples of [ 2 -1 -1 0 ] grain boundaries viewed by TEM along the [2 - 1 -1 0 ] common direction in the W2-50Co alloy sintered for $5 \mathrm{~h}$. The boundary habit plane is a basal (a-b) or prismatic (c) facet parallel to a low-index plane

EBSD measurements of rotation angles and TEM observations indicate the existence of preferred [ $2-1-10]$ boundaries involving the basal or prismatic plane for one grain and a low-index crystal lattice plane for the other grain. The alignment of such planes is associated with misorientation angles that are similar to the experimental data (Fig. 7) (Table 1).

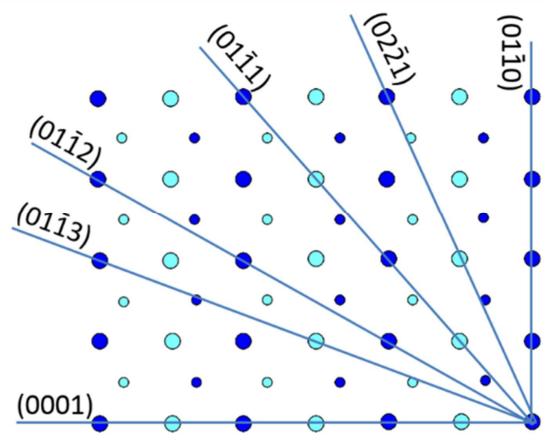

Figure 7. (a) Projection of the WC crystal lattice along [2 - $1-10$ ] showing the distribution of the $(0 \mathrm{~h}-\mathrm{h} \mathrm{I})$ planes. Large and small discs are $\mathrm{W}$ and $\mathrm{C}$ atoms, respectively, at height 0 or $1 / 6\left[\begin{array}{llll}2 & -1 & -1 & 0\end{array}\right]$.

\begin{tabular}{|c|c|c|}
\hline$\mu$ plane & $\lambda$ plane & Angle \\
\hline$\left(\begin{array}{llll}0 & 0 & 0 & 1\end{array}\right)$ & $\left(\begin{array}{llll}0 & 1 & -1 & 3\end{array}\right)$ & $20.59^{\circ}$ \\
\hline 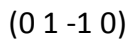 & $\left(\begin{array}{llll}0 & 2 & -2 & 1\end{array}\right)$ & $23.92^{\circ}$ \\
\hline$\left(\begin{array}{llll}0 & 0 & 0 & 1\end{array}\right)$ & $\left(\begin{array}{llll}0 & 1 & -1 & 2\end{array}\right)$ & $29.41^{\circ}$ \\
\hline 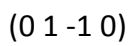 & $\left(\begin{array}{llll}0 & 1 & -1 & 1\end{array}\right)$ & $41.58^{\circ}$ \\
\hline$\left(\begin{array}{llll}0 & 0 & 0 & 1\end{array}\right)$ & $\left(\begin{array}{llll}0 & 1 & -1 & 1\end{array}\right)$ & $48.42^{\circ}$ \\
\hline$\left(\begin{array}{llll}0 & 1 & -1 & 0\end{array}\right)$ & $\left(\begin{array}{llll}0 & 1 & -1 & 2\end{array}\right)$ & $60.59^{\circ}$ \\
\hline$\left(\begin{array}{llll}0 & 0 & 0 & 1\end{array}\right)$ & $\left(\begin{array}{llll}0 & 2 & -2 & 1\end{array}\right)$ & $66.08^{\circ}$ \\
\hline$\left(\begin{array}{llll}0 & 1 & -1 & 0\end{array}\right)$ & $\left(\begin{array}{llll}0 & 1 & -1 & 3\end{array}\right)$ & $69.41^{\circ}$ \\
\hline$\left(\begin{array}{llll}0 & 0 & 0 & 1\end{array}\right)$ & $\left(\begin{array}{llll}0 & 1 & -1 & 0\end{array}\right)$ & $90^{\circ}$ \\
\hline
\end{tabular}

Table 1. List of rotation angles leading to the parallelism of $(0 \mathrm{~h}-\mathrm{h} \mathrm{I})_{\lambda}$ planes with $\left(\begin{array}{llll}0 & 0 & 1\end{array}\right)_{\mu}$ or $\left(\begin{array}{llll}0 & 1 & -1 & 0\end{array}\right)_{\mu}$ planes.

Moreover, some boundaries do not show a perfect alignment between the planes previously outlined. Figure 8 gives examples of such boundaries. In Fig. 8.a, the rotation angle is equal to $59.8^{\circ}$ and in addition, there is a misalignment of the [2 - $1-10$ ] direction of the crystals of about $0.5^{\circ}$. Compared with table 1 , the boundary deviates from the exact $60.59^{\circ}\left(\begin{array}{llll}0 & 1-1\end{array}\right)_{\mu}$ 
// ( $01-12)_{\lambda}$ orientation. In the upper part of the image, the boundary habit plane adopts the $\left(\begin{array}{llll}0 & 1 & -1 & 0\end{array}\right)_{\mu}$ habit plane while in the lower part, the boundary shows successive small facets parallel to $\left(\begin{array}{llll}0 & 1 & -1 & 0\end{array}\right)_{\mu}$ or $\left(\begin{array}{llll}0 & 0 & 0 & 1\end{array}\right)_{\lambda}$ which are close to the $66.08^{\circ}\left(\begin{array}{llll}0 & 0 & 0 & 1\end{array}\right)_{\lambda} / /\left(\begin{array}{llll}0 & 2 & -2 & 1\end{array}\right)_{\mu}$ orientation (table 1). In the example of Fig. $8 \mathrm{~b}$, the rotation angle is $46.3^{\circ}$ and there is a deviation of about $3^{\circ}$ between the [ $\left.2-1-10\right]$ directions of the crystals. The boundary facets are along $\left(\begin{array}{llll}0 & 0 & 0 & 1\end{array}\right)_{\mu}$ and $\left(\begin{array}{llll}0 & 1 & -1 & 0\end{array}\right)_{\lambda}$. The experimental orientation relationship lies between the $41.58^{\circ}\left(\begin{array}{llll}0 & 1 & -1 & 0\end{array}\right)_{\lambda} / /\left(\begin{array}{llll}0 & 1 & -1 & 1\end{array}\right)_{\mu}$ and the $48.42^{\circ}\left(\begin{array}{llll}0 & 0 & 0 & 1\end{array}\right)_{\mu} / /\left(\begin{array}{llll}0 & -1 & 1 & 1\end{array}\right)_{\lambda}$ misorientations (Table 1). The experimental boundary faceting suggests successive adoption of the two configurations through a suitable dislocation network.
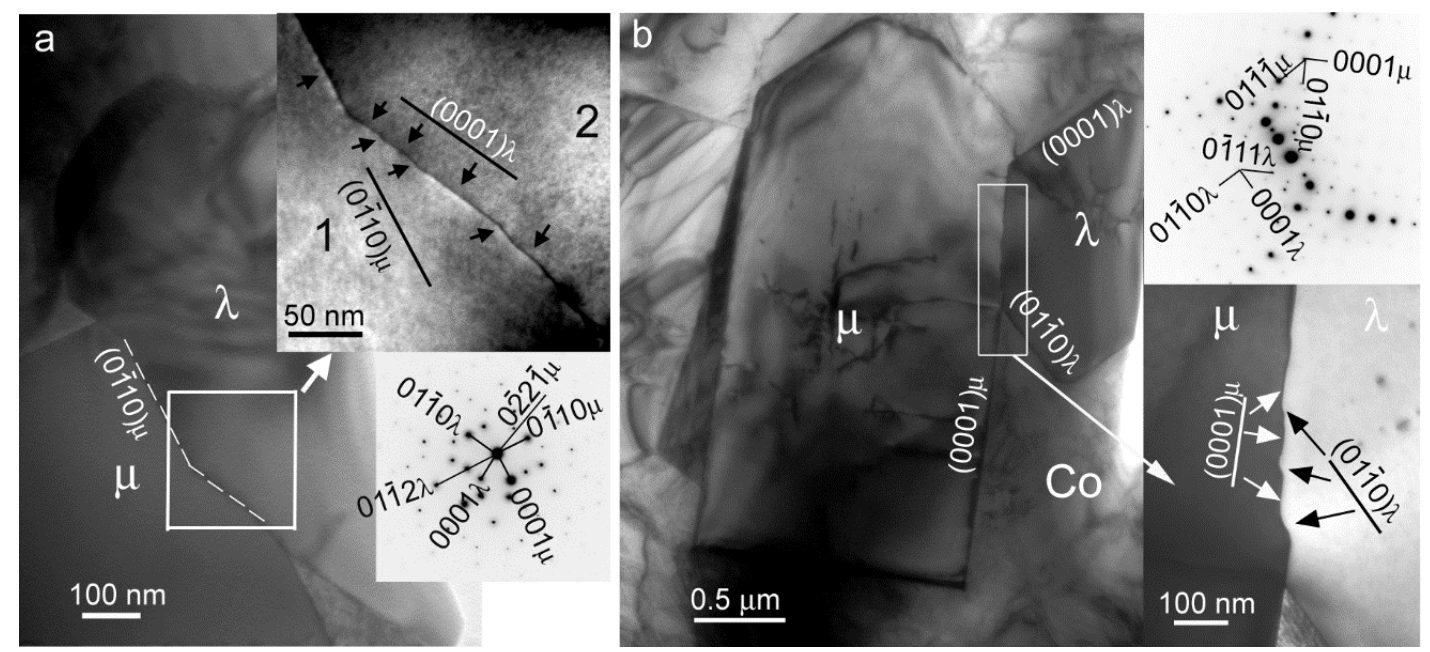

Figure 8. Examples of grain boundaries deviating from preferred misorientations in the W2$50 \mathrm{Co}$ alloy sintered for $5 \mathrm{~h}$. (a) Bright field image of a boundary with a rotation angle of $59.8^{\circ}$, associated diffraction patterns, and magnification of the habit plane. (b) Bright field image of the boundary with a rotation angle of $46.3^{\circ}$ and associated diffraction pattern. The magnification shows faceting of the habit plane.

\subsection{Study of the $26.2^{\circ}[2-1-10]$ boundary}



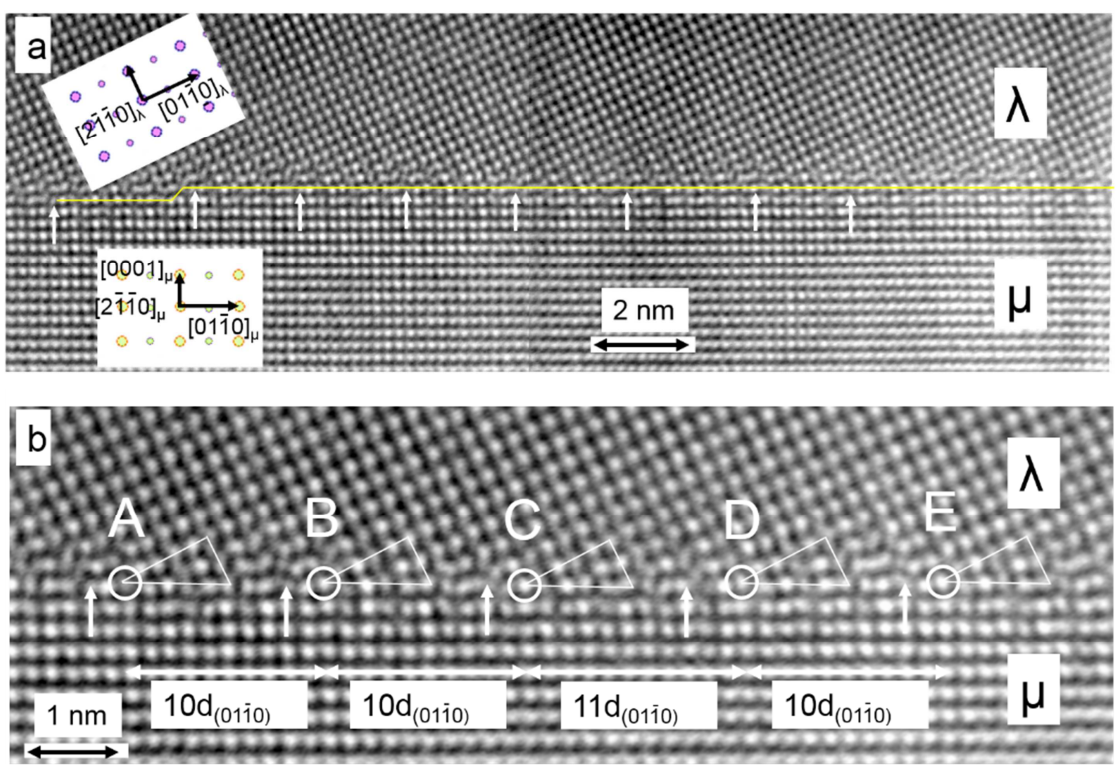

Figure 9. HRTEM images of the $26.2^{\circ}$ [ 2 - $1-10$ ] grain boundary viewed along the [2 -1 -1 0] direction in the WC-20Co, $\mathrm{Cr}, \mathrm{C}$ alloy. (a) A series of atomic planes in the crystal $\mu$ ending at the boundary is observed. (b) Similar atomic patterns are identified along the boundary.

Figure 9 sheds some light on the atomic structure of a [ 2 - $1-1$ - 0 ] asymmetrical tilt boundary found in the WC-20Co, $\mathrm{Cr}, \mathrm{C}$ alloy. The misorientation angle is close to $26.2^{\circ}$, and the habit plane deviates from the exact parallelism between low index planes. The boundary plane is parallel to the basal plane for one crystal and deviates by about $3^{\circ}$ from the $(01-12)$ plane for the other crystal. The boundary is close to the $29.41^{\circ}\left[\begin{array}{lll}2 & -1 & -1\end{array}\right]$ ] orientation with $(000$ $1)_{\mu}(01-12)_{\lambda}$ as habit plane (table 1$)$.

In the experimental boundary, some $\left(\begin{array}{llll}0 & 1 & -1 & 0\end{array}\right)_{\mu}$ atomic planes terminating at the boundary in the lower crystal are observed with a mean spacing of about $2.5 \mathrm{~nm}$ (Fig. 9a). More careful examination of the boundary reveals other similar atomic patterns repeating along the boundary, arbitrarily pointed out as triangles on the image (Fig. 9b). Such atomic arrangements were also observed for asymmetrical CSL boundaries in cubic or hexagonal crystals $[20,21]$ (Fig. 9b). They indicate the existence of periodic atom units, specific to the boundary structure [22-24]. A deeper analysis using atomistic modelling would be necessary to study the features of specific structural units in this tilt boundary. In the present work, a geometrical approach is used, considering that the grain boundary deviates from a reference boundary $[25,26]$. In this approach, the deviation is accommodated by a network of dislocations whose characteristics depend on the grain boundary misorientation [27]. This description has a link with the concept of atomic structural units as the latter are associated with intrinsic dislocations [22-24,28].

Crystallographically equivalent points, designated as $A$ to $E$ on the figure, are identified along the boundary. The interval between these points is equal to $10 \mathrm{~d}_{01-10}(2.5 \mathrm{~nm})$, where $\mathrm{d}_{01-10}$ is the spacing between $(01-10)$ atomic planes, except between $C$ and $D$ where it is equal to $11 \mathrm{~d}_{01-10}$. For the exact $\left(\begin{array}{llll}0 & 0 & 0 & 1\end{array}\right)_{\mu}\left(\begin{array}{llll}0 & 1 & -1 & 2\end{array}\right)_{\lambda}$ boundary, a large mismatch of $\varepsilon=13.8 \%$ is calculated with $\varepsilon=2\left(d_{\lambda}-d_{\mu}\right) /\left(d_{\lambda}+d_{\mu}\right)$ where $d_{\lambda}$ and $d_{\mu}$ are the magnitudes of $1 / 2[01-1-1]_{\lambda}$ and 
$1 / 2\left[\begin{array}{lll}0 & 1 & -1\end{array}\right]_{\mu}$, respectively (Fig. 10). At the boundary, the lattices almost coincide every $16 d_{01-}$ 10 planes $(4.0 \mathrm{~nm})$ and, in between, two $(01-10)_{\mu}$ planes are expected to stop to compensate for the misfit. In the middle of the period, lattice nodes nearly coincide within $1 / 6[2-1-10]$. This configuration should lead to a periodic array of misfit dislocations with a spacing of $8 \mathrm{~d}_{01-10}$ planes $(2.0 \mathrm{~nm})$. As the experimental boundary deviates by a few degrees from this orientation, dislocations with an edge component perpendicular to the habit plane are also expected to be found in the boundary.

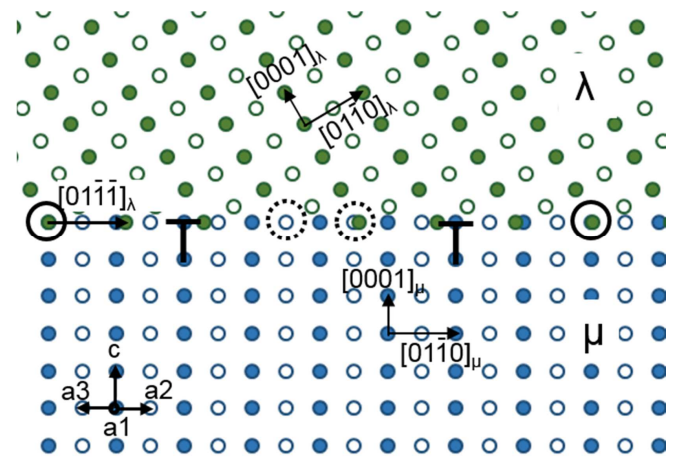

Figure 10. Schematic diagram of the $29.41^{\circ}$ [ 2 - 1 -1 0 ] grain boundary viewed along [2 - $1-10$ ] direction. The mismatch between $\left[\begin{array}{llll}0 & 1 & -1 & 0\end{array}\right]_{\mu}$ and $\left[\begin{array}{lll}0 & -1 & -1\end{array}\right]_{\lambda}$ is equal to $13.8 \%$. Only atomic positions of $\mathrm{W}$ atoms (or equivalently $\mathrm{C}$ atoms) are represented on the drawing and empty discs are out of the projection plane ( $\pm 1 / 6[2-1-10])$. The solid circles indicate close coincidence of the atom sites at the boundary and dotted circles a difference in height of $1 / 6[2-1-10]$. Basic lattice vectors ai ( $i=1$ to 3 ) and $c$ are drawn in the crystal $\mu$ (a1 $=1 / 3[2-1$ $-10], a 2=1 / 3\left[\begin{array}{llll}-1 & 2 & -1 & 0\end{array}\right], a 3=1 / 3\left[\begin{array}{llll}-1 & -1 & 2 & 0\end{array}\right]$ and $\left.c=\left[\begin{array}{llll}0 & 0 & 0 & 1\end{array}\right]\right)$.

\subsubsection{Method}

In order to study the dislocations lying in the experimental $26.2^{\circ}$ boundary, a reference boundary was defined according to an approach developed for mismatched boundaries $[25,26]$. This reference boundary is chosen to force coherency at the boundary. In what follows, a modified $29.41^{\circ}$ boundary is used as the coherent reference boundary. The lower crystal is stretched in order to coincide with the upper crystal in agreement with the presence of misfit dislocations in the experimental boundary (Figure 9). In the boundary plane, the [ $2-1-10]$ direction is common to the crystals, and is kept unchanged, only the [0 $\left.\begin{array}{lll}1 & -1 & 0\end{array}\right]_{\mu}$ vector is stretched in such a way that $\left[\begin{array}{llll}0 & 1 & -1 & 0\end{array}\right]_{\mu}=\left[\begin{array}{llll}0 & 1 & -1 & -1\end{array}\right]_{\lambda}$. The $26.2^{\circ}$ experimental boundary is assumed to deviate from this reference boundary. The interface dislocations accommodating the parametric misfit and the angular deviation are characterized by comparison with the reference boundary.

As an example, Fig. 11 depicts the boundary between points $B$ and $C$ of figure 9 . The Burgers vector $b$ associated with the whole period is determined using the circuit mapping procedure [29]. Two half-circuits $C_{\lambda}$ and $C_{\mu}$ are drawn in crystals $\lambda$ and $\mu$, starting and finishing at the interface, at equivalent points. The same circuit is drawn in the dichromatic pattern that consists of $\lambda$ and stretched $\mu$ interpenetrated lattices, to image the closure 
failure regarding the reference boundary. The Burgers vector in the coordinate system of the $\lambda$ crystal is equal to :

$$
\begin{gathered}
\quad b=-\left(C_{\lambda}+P_{\text {coh }} C_{\mu}\right) \\
\text { with } \quad P_{\text {coh }}=S^{-1} R^{-1} D S
\end{gathered}
$$

where the transformation $P_{\text {coh }}$ expresses a vector of lattice $\mu$ in the coordinate system of lattice $\lambda$ in the coherent reference boundary. $P_{\text {coh }}$ depends on the rotation $R$ relating the crystals and on the deformation $D$ applied to $\mu$ crystal [25-26]. In the present case, the orientation relationship of the reference boundary is $\theta=29.406^{\circ}$ around the common [ $2-1$ $10]$ axis, and the deformation is an expansion of $e=1.148$ parallel to the $\left[\begin{array}{lll}0 & 1 & -1\end{array}\right]_{\mu}$ direction. The vectors of the $\lambda$ and $\mu$ crystals were expressed in Miller indices for matrix operations and the structure matrix $S$ was used to express the vectors in the orthonormal

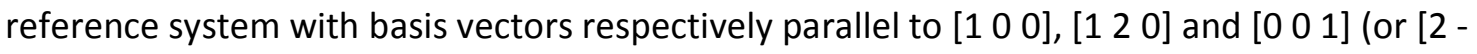
$\left.\begin{array}{lll}1 & -1 & 0\end{array}\right],\left[\begin{array}{llll}0 & 1 & -1 & 0\end{array}\right]$ and [ $\left.\begin{array}{llll}0 & 0 & 0 & 1\end{array}\right]$ in 4 Miller Bravais indices). So, R, D and S, expressed in the orthonormal coordinate system, are:

$\mathrm{R}=\left(\begin{array}{ccc}1 & 0 & 0 \\ 0 & \cos (\theta) & -\sin (\theta) \\ 0 & \sin (\theta) & \cos (\theta)\end{array}\right) \quad \mathrm{D}=\left(\begin{array}{ccc}1 & 0 & 0 \\ 0 & \mathrm{e} & 0 \\ 0 & 0 & 1\end{array}\right) \quad \mathrm{S}=\left(\begin{array}{ccc}a & -a / 2 & 0 \\ 0 & \mathrm{a} \sqrt{3} / 2 & 0 \\ 0 & 0 & \mathrm{c}\end{array}\right)$

with $\mathrm{a}=0.2906 \mathrm{~nm}$ and $\mathrm{c}=0.2837 \mathrm{~nm}$, lattice parameters of WC

The vector $\mathrm{b}$ can be calculated in the coordinate system of lattice $\mu$ by the $\mathrm{P}_{\text {coh }^{-1}}$ transformation.

For the sake of clarity, the Burgers vectors will be finally expressed as a combination of

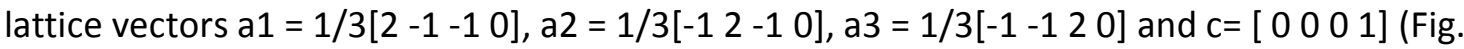
10).

\subsubsection{Characterization of the dislocations}

The procedure described above was applied to determine the Burgers content of the $\mathrm{BC}$ facet (Fig. 11). The Burgers vector is found to be equal to $-a 2_{\mu}$ (or $\left.a 3_{\mu}\right)+1 / 2 c_{\lambda}$ (Table 2). Its component parallel to the [2-1 -1 0] axis is related to the difference in height between $B$ and C along [ $2-1-10]$. Further analysis was conducted along the $\mathrm{BC}$ facet to thoroughly determine the distribution of dislocations in this part of boundary. The issue of core localization for dislocations with such a narrow separation will be discussed after analysis of the boundary. 


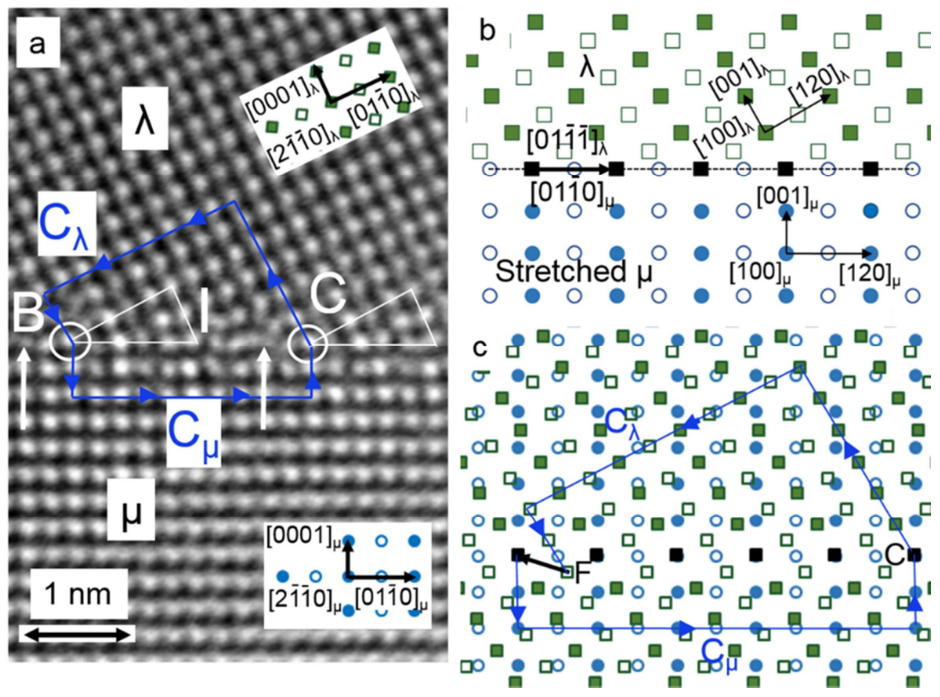

Figure 11. Magnification of the boundary in the BC region. (a) HRTEM image with a Burgers circuit drawn around points $B$ and $C$, (b) schematic diagram of the reference boundary with stretched $\mu$ crystal and lattice vectors expressed in Miller indices, (c) same Burgers circuit as in (a) drawn in the dichromatic pattern associated with the reference boundary.

The Burgers vector associated with the IC part of the boundary is first determined (Fig. $12 a, b)$ (Table 2 ). It can be expressed as a lattice vector of $\mu$. It is equal to $b_{1}=-a 2_{\mu}$ (or $a 3_{\mu}$ ). The dislocation content associated with the $\mathrm{BI}$ fraction of the boundary was determined similarly as $b_{2}=1 / 2 c_{\lambda}$ (Fig. 12a,c).
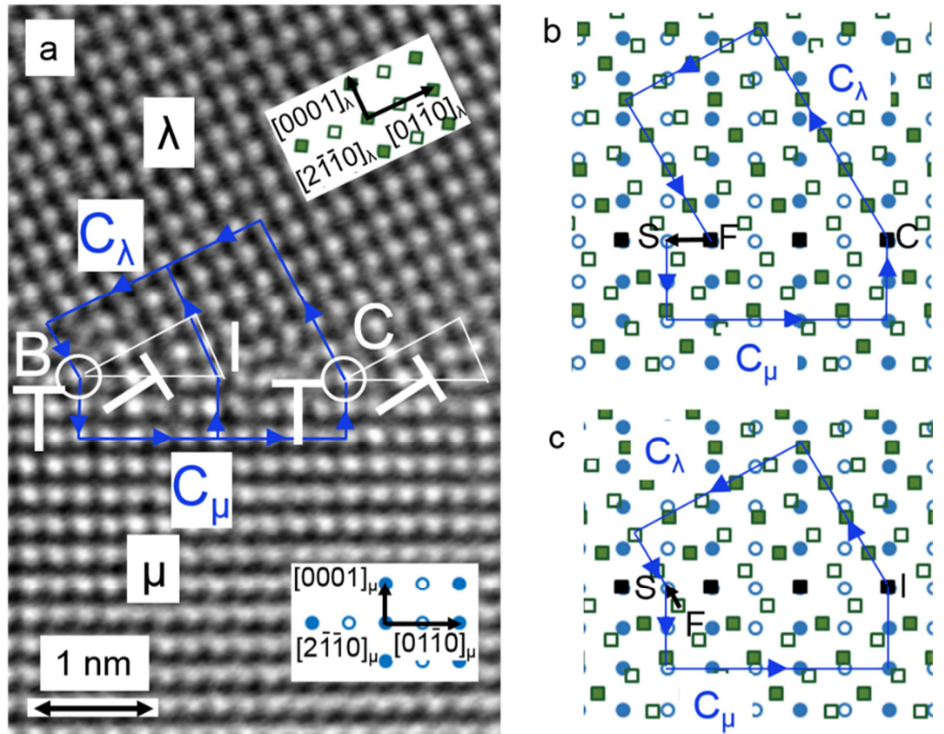

Figure 12. Magnification of the boundary in the BC region. (a) HRTEM image with two Burgers circuits drawn around IC and BI portions of the boundary, (b-c) same Burgers circuits drawn in the dichromatic pattern associated with the reference boundary. 
The Burgers vector associated with the $C D$ part of the boundary, which is associated with a period of $11 d_{01-10}$, was also identified (Fig. 13). It is found to be equal to $-a 2_{\mu}$ (or $a 3_{\mu}$ ) $+c_{\lambda}$ (or $\left.b_{1}+2 b_{2}\right)$. It is different from BC facet. Further analysis was conducted for comparison with $B C$. The dislocation content associated with the JD fraction is a lattice vector of $\lambda,(-a 2+c)_{\lambda}$ (or $\left.(a 3+c)_{\lambda}\right)$ or the sum $-a 2_{\mu}+1 / 2 c_{\lambda}$ (or $\left.a 3_{\mu}+1 / 2 c_{\lambda}\right)\left(\right.$ or $\left.b_{1}+b_{2}\right)$. The JD portion has a larger Burgers vector than that of IC. The difference with IC is $b_{2}=1 / 2 C_{\lambda}$. The Burgers vector associated with the triangular pattern in the $\mathrm{CJ}$ part of the boundary is equal to $\mathrm{b}_{2}$, as for $\mathrm{BI}$.
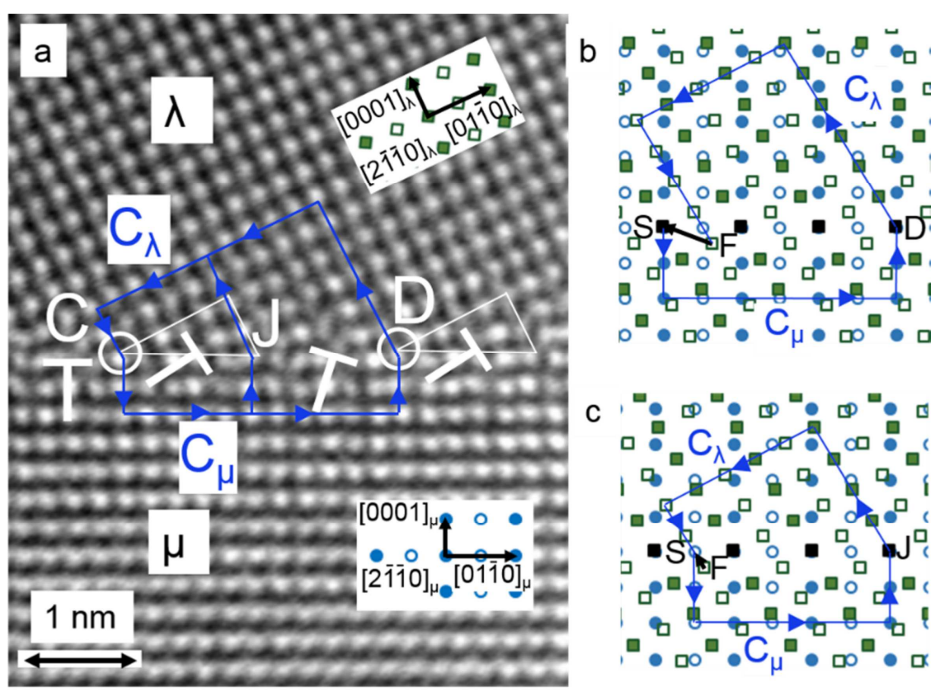

Figure 13. Study of the boundary in the C-D region. (a) HRTEM image with two Burgers circuits drawn around the JD and CJ portions of the boundary, $(b-c)$ same Burgers circuits drawn in the dichromatic pattern associated with the reference boundary.

\begin{tabular}{|c|c|c|c|c|c|c|}
\hline & $C_{\lambda}$ & $\mathrm{C}_{\mu}$ & $\begin{array}{c}\mathrm{C}_{\mu} \\
\text { expressed } \\
\text { in } \lambda\end{array}$ & $b_{\lambda}$ & $b_{\mu}$ & $\mathrm{b}$ \\
\hline BC & $\begin{array}{l}\frac{1}{2}\left[\begin{array}{lll}-9 & -18 & 8\end{array}\right] \\
\pm \frac{1}{2}\left[\begin{array}{lll}1 & 0 & 0\end{array}\right]\end{array}$ & {$\left[\begin{array}{lll}5 & 10 & 0\end{array}\right]$} & $5\left[\begin{array}{lll}1 & 2 & -1\end{array}\right]$ & $\begin{array}{l}{\left[\begin{array}{lll}0 & -1 & 1\end{array}\right]} \\
\left.\text { (or }\left[\begin{array}{lll}-1 & -1 & 1\end{array}\right]\right)\end{array}$ & $\sim\left[\begin{array}{llll}-0.12 & -1.24 & 0.43\end{array}\right]$ & $\begin{array}{l}-a 2_{\mu}\left(\text { or } a 3_{\mu}\right)+\frac{1}{2} c_{\lambda} \\
\left(b_{1}+b_{2}\right)\end{array}$ \\
\hline IC & {$\left[\begin{array}{lll}-2 & -4 & 2\end{array}\right]$} & $\begin{array}{l}\frac{5}{2}\left[\begin{array}{lll}1 & 2 & 0\end{array}\right] \\
\pm \frac{1}{2}\left[\begin{array}{lll}1 & 0 & 0\end{array}\right]\end{array}$ & $\begin{array}{l}{\left[\begin{array}{lll}3 & 5 & -2\end{array}\right]} \\
\text { (or } \\
\left.\left[\begin{array}{lll}2 & 5 & -2\end{array}\right]\right)\end{array}$ & $\begin{array}{l}\frac{1}{2}[-2-21] \\
\left(\text { or } \frac{1}{2}\left[\begin{array}{lll}0 & -2 & 1\end{array}\right]\right)\end{array}$ & $[-1-10]$ (or $[0-10])$ & $\begin{array}{l}-a 2_{\mu}\left(\text { or } a 3_{\mu}\right) \\
\left(b_{1}\right)\end{array}$ \\
\hline $\begin{array}{l}\mathrm{BI} \\
\text { or } \\
\mathrm{CJ}\end{array}$ & $\begin{array}{l}\frac{1}{2}\left[\begin{array}{lll}-5 & -10 & 4\end{array}\right] \\
\pm \frac{1}{2}\left[\begin{array}{lll}1 & 0 & 0\end{array}\right]\end{array}$ & $\begin{array}{l}\frac{5}{2}\left[\begin{array}{lll}1 & 2 & 0\end{array}\right] \\
\pm \frac{1}{2}\left[\begin{array}{lll}1 & 0 & 0\end{array}\right]\end{array}$ & $\frac{5}{2}\left[\begin{array}{lll}1 & 2 & -1\end{array}\right]$ & $+\frac{1}{2}\left[\begin{array}{lll}0 & 0 & 1\end{array}\right]$ & $\sim\left[\begin{array}{llll}-0.12 & -0.24 & 0.43\end{array}\right]$ & $\begin{array}{l}\frac{1}{2} c_{\lambda} \\
\left(b_{2}\right)\end{array}$ \\
\hline
\end{tabular}

Table 2. Determination of the Burgers vectors expressed in the $\lambda$ and $\mu$ crystals. Miller indices were used for intermediate calculations.

The validity of the result was checked by simulating the elastic displacement field around the dislocations in the framework of the linear elasticity theory, using the HRPACK software [30]. The relative displacements at the boundary for a periodic array of misfit dislocations are represented by a sawtooth function, with no deformation at the midpoint between dislocation cores and progressive deformation towards the cores [31]. The previous analysis of the boundary has revealed periodic pairs of dislocations with mainly Burgers vectors equal 
to $b_{1}=-a 2_{\mu}\left(\right.$ or $\left.a 3_{\mu}\right)$ and $b_{2}=1 / 2 c_{\lambda}$, with a periodicity of $2.5 \mathrm{~nm}$. Only one array of periodic misfit dislocations can be handled by the software, so the calculation covered a periodic series with Burgers vector equal to $b_{1}+b_{2}$, with a periodicity of $2.5 \mathrm{~nm}$. The core was positioned where the $b_{1}$ dislocation was found. The calculation indicates that almost no deformation is expected around the dislocation core (Fig. 14). The calculated positions of the atomic columns are in good agreement with the experimental image except very close to the dislocation core where the calculation is not valid. At the boundary, some further atomic relaxations are expected independently of the dislocation content. This simulation confirms the above determination of the Burgers vectors. The simulation of Fig. 14, where both dislocations are combined, also raises questions about the existence of two separate cores given the small distance ( $1 \mathrm{~nm}$ ) between $b_{1}$ and $b_{2}$ dislocations. It is tricky to answer this question from the calculated elastic displacement field, as each dislocation causes virtually no deformation around its core. On the other hand, two dislocations were found by the circuit mapping procedure. In what follows, the existence of two sets of dislocations is therefore assumed. However, the main conclusions on the atomic arrangement at this mismatched boundary would be valid by assuming delocalized dislocation cores.

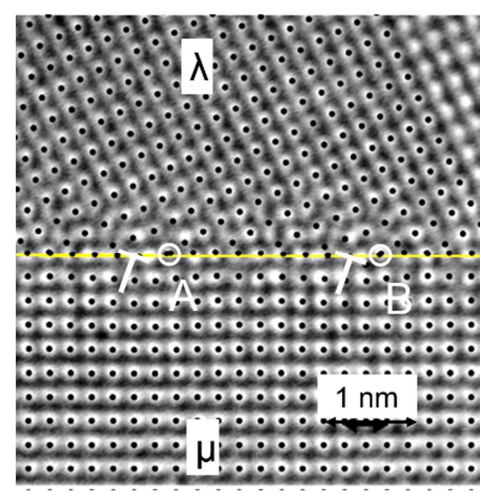

Figure 14. Study of the boundary in the A-B region. The elastic displacement field calculated for a periodic array of $-a 2_{\mu}$ (or $\left.a 3_{\mu}\right)+1 / 2 C_{\lambda}$ misfit dislocations is superimposed on the HRTEM image.

\subsubsection{Characteristics of the dislocations}

The presence of dislocations with a Burgers vector equal to a crystal lattice vector, like $b_{1}=-$ $\mathrm{a} 2_{\mu}$ (or $\mathrm{a} 3_{\mu}$ ) dislocations along boundaries, is not common as the elastic energy of a dislocation varies with respect to $b^{2}$ [32]. Boundary dislocations are usually associated with smaller Burgers vectors belonging to the Displacement Shift complete lattice (DSC) [33]. These vectors relate lattice nodes of the two crystals in the dichromatic pattern (Fig 15). The $b_{2}=1 / 2 c_{\lambda}$ vector is one of the smallest DSC lattice vectors. Most dislocations along a boundary lead to the formation of a step, the height of which can be predicted if the boundary structure is the same on each side of the step [34,35]. The presence of a step along a boundary is of importance as the dislocation energy is the balance between core and step energy. In the part of the boundary observed by HRTEM (Fig. 9), the habit plane looks flat, no step is visible except on the left part of figure 9. When the Burgers vector is a lattice vector, no step is expected, as observed for the dislocation in the IC or JD part of the boundary. Conversely, a step is expected for non-lattice dislocations such as the $1 / 2 c_{\lambda}$ 
dislocation in the $\mathrm{BI}$ or $\mathrm{CJ}$ part. These dislocations with step character are called disconnections [36]. The step height can be determined geometrically by defining a step vector in each crystal, $S_{\lambda}$ and $S_{\mu}$ with $b=S_{\lambda}-S_{\mu}[34,35]$. The step height in each crystal is equal to the magnitude of the step vector along the normal of the grain boundary. For the $1 / 2 c_{\lambda}$ disconnection, the height is equal to zero in the $\mu$ crystal and to $1 \mathrm{~d}_{(01-12)}(=0.12 \mathrm{~nm})$ in

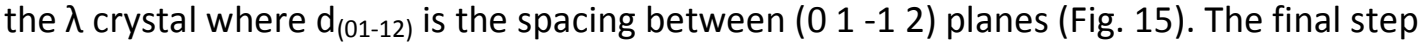
height is the mean value of the step heights in the two crystals, leading to an average value of $0.06 \mathrm{~nm}$. The small magnitude of the step height and strain associated with the $1 / 2 C_{\lambda}$ disconnection probably explains why the step is hardly visible on the HRTEM image. The steps therefore make a limited contribution in the boundary area which is favourable in terms of grain boundary stability [37].

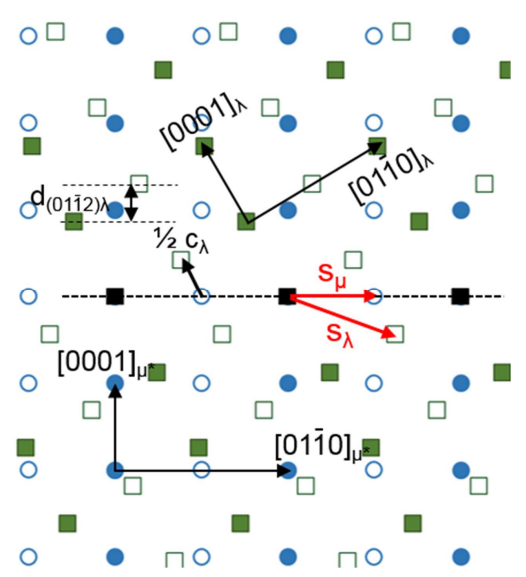

Figure 15: Dichromatic pattern associated with the reference boundary depicting step vectors attached to the $1 / 2 \mathrm{C}_{\lambda}$ disconnection found in this work.

\subsubsection{Interpretation of the dislocation array}

The dislocation network found in the boundary is interpreted by considering the parametric mismatch at the boundary and the angular deviation of $3.2^{\circ}$ from the coherent $29.41^{\circ}$ [2 - 1 $10]$ ] reference boundary. Two arrays of dislocations were observed in the boundary $b_{1}=-a 2_{\mu}$ (or $a 3_{\mu}$ ) dislocations and $b_{2}=1 / 2 c_{\lambda}$ disconnections, with a periodicity close to $2.5 \mathrm{~nm}$. The dislocation lines are parallel to [ $\left[\begin{array}{lll}2 & -1 & -1\end{array}\right]$.

The $-a 2_{\mu}$ and $a 3_{\mu}$ vectors are parallel to the boundary, with an edge component equal to

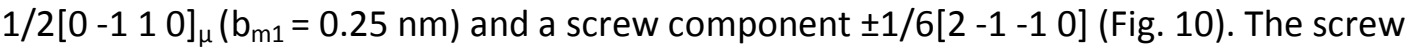
component does not participate in accommodating the deviation from the reference boundary. The energy of the dislocation array can be minimised if the dislocations adopt alternatively the $-a 2_{\mu}$ and $a 3_{\mu}$ vector with opposite screw $1 / 6[2-1-10]$ components. The $1 / 2 c_{\lambda}$ disconnections have a Burgers vector component parallel to $\left[\begin{array}{lll}0 & -1 & 1\end{array}\right]_{\mu}\left(b_{\mathrm{m} 2}=0.07 \mathrm{~nm}\right)$ and a component perpendicular to the boundary plane $\left(b_{e}=0.12 \mathrm{~nm}\right)$ (Fig. 10).

The $b_{m 1}$ and $b_{m 2}$ components of the $-a 2_{\mu}\left(a 3_{\mu}\right)$ and $1 / 2 c_{\lambda}$ dislocations accommodate the parametric mismatch and the $b_{e}$ component of the $1 / 2 c_{\lambda}$ dislocations accommodates the tilt rotation. The exact parametric mismatch between the crystals depends on the angular deviation from $29.41^{\circ}$. In what follows, the 02 -lattice approach is used to calculate the 
dislocation array characteristics necessary to compensate for the deviation from the reference boundary using the experimentally found Burgers vectors [27]. The deviation A from this preferred state is the product of the deformation $D$ relating $\mu$ and stretched $\mu$ lattices by the small angle rotation $\mathrm{Rd}$ describing the deviation from $29.41^{\circ}$ [ $2-1-10$ ] orientation (Figure 16a):

$A=R d^{*} D$

where $D$ is the expansion described in (3) and Rd is a rotation of $\alpha$ angle around the common [ $2-1-10]$ axis expressed in the orthonormal system of the $\mu$ crystal :

$\mathrm{Rd}=\left(\begin{array}{ccc}1 & 0 & 0 \\ 0 & \cos (\alpha) & -\sin (\alpha) \\ 0 & \sin (\alpha) & \cos (\alpha)\end{array}\right)$

The dislocation network is deduced from the 02-lattice equation:

$\left(I-A^{-1}\right) X^{02}=b$ or $\quad T X^{02}=b$

where $I$ is the unit transformation and $b$ is the Burgers vector of a set of boundary dislocations. $\mathrm{X}^{02}$ vectors, define 02-elements which are determined for each Burgers vector. 02-elements form the 02-lattice which can be a point lattice, or consist of parallel lines or parallel planes, defining the places of best match between the lattices of both crystals. Dislocation walls are located in the form of cells around the 02-elements. The dislocation lines are located at the intersection of the dislocation walls with the boundary plane.

The experimental misorientation angle is $26.2^{\circ}$ compared with $29.41^{\circ}$, so the equation was solved for $\alpha$ angles ranging from 0 to $-5^{\circ}$ using the two Burgers vectors identified in the boundary $b_{1}$ and $b_{2}$. For the sake of accuracy, the $R d^{*} b_{2}$ vector was used instead of $b_{2}$ to take into account the $R d$ rotation. When $\alpha$ is equal to 0 , the deviation is a pure expansion, the rank of the T matrix is equal to 1 , the 02 -lattice elements are planes perpendicular to the

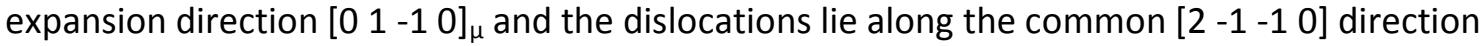
in the boundary plane. When $\alpha$ is different from 0 , the rank of the T matrix is equal to 2 , the 02-lattice elements are lines parallel to the [ $2-1-10]$ common axis, and the dislocations also lie along the common [2 - $-1-10$ ] direction in the boundary plane. Their spacing is deduced from the $\mathrm{X}^{02}$ vectors coordinates (Fig. 16b). If there is no angular deviation, only $b_{1}$ dislocations are needed in the boundary and the calculated dislocation spacing is equal to $1.95 \mathrm{~nm}$. If there is a deviation, $b_{2}$ dislocations appear and their spacing decreases when the absolute value of the deviation angle increases while the spacing of $b_{1}$ dislocations slightly increases. The results are in agreement with the above analysis of the dislocation character showing that $b_{1}$ dislocations accommodate the mismatch at the boundary and $b_{2}$ dislocations accommodate the angular deviation and a part of the parametric mismatch. The calculated spacings for $b_{1}$ and $b_{2}$ dislocations at the observed misorientation are equal to 2.4 and $2.3 \mathrm{~nm}$ in close agreement with the mean experimental value $(2.5 \mathrm{~nm})$. Note that this calculation was performed using the $\left(b_{1}, b_{2}\right)$ dislocation pair ( $B C$ period). The other observed pair of dislocations (CD part of the boundary) has a larger edge character. Such periodically spaced dislocations could provide an additional accommodation of the tilt deviation and parametric mismatch what could explain the slightly larger experimental dislocation spacing compared to the calculation. 

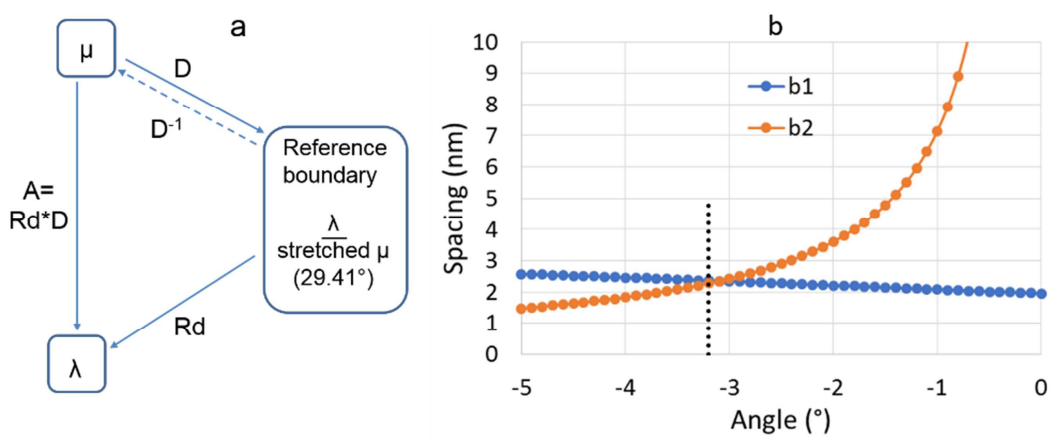

Figure 16. (a) Diagram describing the deviation of the experimental $26.2^{\circ}$ [2 - $1-10$ ] boundary from the reference boundary as defined in §3.3.1. (b) Dislocation spacing associated with $b_{1}$ and $b_{2}$ Burgers vectors as a function of the deviation angle. The dotted line corresponds to the observed boundary misorientation.

The array of boundary dislocations is associated with an elastic energy that depends on the dislocation character and spacing, and as such it evolves as a function of the deviation angle. In the present case, the two sets of dislocations are not independent in elasticity so the calculation of their energy is complex [38]. However, the increase in the $b_{1}$ dislocation spacing when the absolute value of the angle increases could result in an energy minimum for a given tilt angle. In this assumption, the most stable boundary would not correspond to $\left(\begin{array}{llll}0 & 0 & 0 & 1\end{array}\right)$ and $\left(\begin{array}{llll}0 & 1 & -1 & 2\end{array}\right)$ planes exactly parallel but slightly deviating planes.

\section{Discussion}

The fraction of [2 -1 -1 0] boundaries in WC-Co alloys increases with Co content, probably due to a higher mobility of WC grains at high temperature in a larger amount of binder and to their lower energy compared with random boundaries. No change in the [2 -1 - 10$]$ boundary fraction was found between $1 \mathrm{~h}$ and $5 \mathrm{~h}$ sintering time which indicates an optimisation of the grain boundary population in the WC-Co alloys already after $1 \mathrm{~h}$ of sintering. An effect of carbon content was expected owing to small differences in interface and boundary energy [14]. This study shows that it has no significant impact on the amount of [2 -1 -1 0] boundaries. The stability of these grain boundaries is probably related to the nature of the habit plane, which is always parallel to a basal or prismatic plane of one of the adjacent grains, and a low index plane of the other grain, as previously observed in metals [39] or in ceramics [40]. The basal and prismatic planes are low energy habit planes for the WC grains in WC-Co alloys [14] what probably diminishes the energy of [2 -1 -1 0] boundaries.

Specific [ $2-1-10$ ] boundaries are identified. The occurrence of special orientations is usually related to the good coherency of the crystal lattices meeting at the boundary and to a cusp in energy. For [ $2-1-10$ ] boundaries, there is an exact match along the [2 -1 -1 0] axis, but there is no rational match along the perpendicular direction in the boundary. The lattice coherency is examined for frequently observed boundaries (Fig. 17). Assuming a tiny mismatch, coincident crystal lattice nodes are found for the $60.59^{\circ}$ misorientation $(\Sigma=4$ 
boundary). Looking at the $\mathrm{W}$ lattice of the crystals, one $\mathrm{W}$ atom site in two is common at the boundary habit plane. Atomistic calculations performed for this boundary have shown that it is associated with a relatively low energy $[14,41]$. The $48.42^{\circ}, 90^{\circ}$ and $29.41^{\circ}$ boundaries contain a lower density of common lattice nodes: the period between near coincidence sites is equal to $0.6 \mathrm{~nm}$ for $60.59^{\circ}, 1.5 \mathrm{~nm}$ for $48.42^{\circ}, 2.3 \mathrm{~nm}$ for $90^{\circ}$ and $4.0 \mathrm{~nm}$ for $29.41^{\circ}$. The smallest mismatch arises for the $60.59^{\circ}$ boundary and the largest for the $48.42^{\circ}$ boundary (Fig. 17).

Although the $26.2^{\circ}$ [ $2-1-10$ ] boundary is associated with a large mismatch, the HRTEM study has revealed a well-organized atomic structure consisting of a periodic arrangement of mainly two kinds of boundary dislocations. These dislocations could be interpreted using a reference boundary with forced coherency [25]. It is suggested that the defect content in the boundary releases the strain due to a parametric misfit and tilt deviation from the $29.41^{\circ}$ [ 2 $1-10$ ] boundary. This $29.41^{\circ}$ [ $2-1-10$ ] orientation leading to the parallelism of the lowindex planes $\left(\begin{array}{llll}0 & 0 & 0 & 1\end{array}\right)$ and $\left(\begin{array}{llll}0 & 1 & -1 & 2\end{array}\right)$ at the boundary can be considered as one of the preferred orientations in cemented carbides.

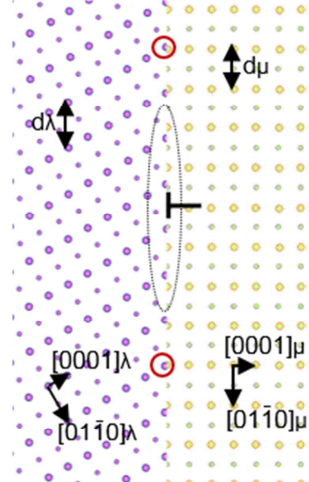

$29.41^{\circ}$

a $\varepsilon=13.8 \%$

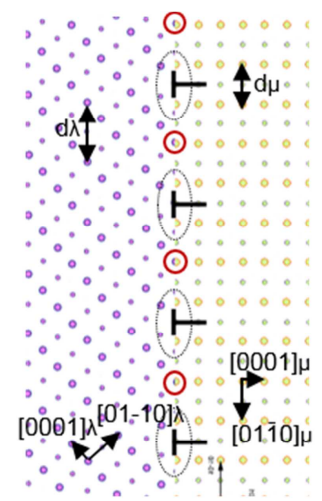

$48.42^{\circ}$

b $(0111) \lambda(0001) \mu$ $\varepsilon=28,1 \%$

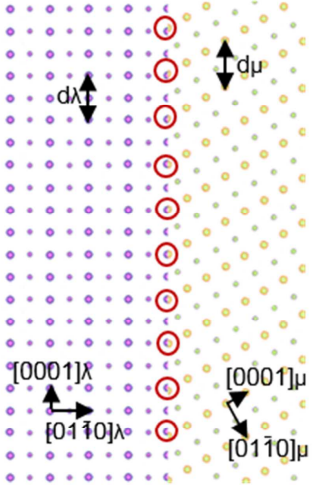

$60.59^{\circ}$

C $\varepsilon=1.8 \%$

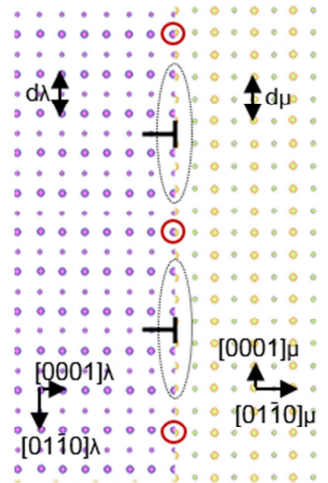

$90^{\circ}$

d $\quad(0001) \lambda(01 \overline{1} 0) \mu$ $\varepsilon=12.0 \%$

Figure 17. Projection of $\mathrm{W}$ lattices along the [ 2 -1 -1 0] direction for several observed boundary misorientations, where large and small disks correspond to height 0 and $1 / 2$, respectively. Red circles represent near coincident sites at the boundary. The values $d_{\lambda}$ and $d_{\mu}$ are used to calculate the misfit between the planes meeting at the boundary.

In this family of boundaries, the $\sim 60^{\circ}[2-1-10]$ boundary $(\Sigma=4)$ is already present in the powder. It could be related to the existence of $\Sigma=2$ grain boundaries in the powder. Assuming a central WC grain and $\Sigma=2$ grain boundaries on two different prismatic facets, this leads to a $60^{\circ}$ [ $2-1-1$ 0] orientation relationship for the two neighboring grains [42]. The high frequency of $\Sigma=4$ boundaries in the sintered alloy indicates that they are resistant to binder infiltration, in agreement with calculations [14]. The grain boundary distribution in the alloys reveals that many [ $2-1-10$ ] grain boundaries are formed during the sintering stage, although the mismatch at the interface is rather high. Moreover, boundaries are not formed strictly parallel to low index crystal planes, and they also deviate from these preferred orientations, owing to suitable dislocation and disconnection arrays in the 
boundaries. In some cases, the formation of facets with two different habit planes is an alternative for the boundary to decrease its energy. However, not all [2 -1 -1 0] boundaries are stable, and only some orientations are favoured, as observed in the angle distribution.

\section{Conclusions}

The observations described in this paper reveal the large number of [ $2-1-10$ ] boundaries in WC-Co alloys. No effect of carbon content or sintering time on the frequency of these boundaries was detected. The number of these boundaries increases with binder content, showing their stability in the material. Several preferred orientations involving the basal or prismatic habit plane were identified leading to the formation of mismatched asymmetrical tilt boundaries. Some boundaries show an angular deviation of several degrees from these special orientations. Periodic arrays of dislocations compensate for the parametric mismatch and angular deviation at these boundaries. [ $2-1-10$ ] boundaries represent between 5 and $10 \%$ of the boundaries in the material and some of them exist in the powder. They probably influence the sintering process, especially the WC skeleton formation as well as the mechanical properties of the alloy, and should be considered for any microstructure modelling. More generally, this study emphasizes the existence of stable boundaries with large mismatch owing to low energy boundary habit planes. This is one step in the understanding of general boundaries which are usually the most numerous in a material, especially after sintering.

\section{Acknowledgments}

The authors would like to thank S. Norgren, J. Angseryd, E. Coronel and T. Persson from Sandvik Mining R\&D Rock Tools, Sandvik Coromant R\&D and Seco Tools AB for fruitful collaboration. R. Bonnet is acknowledged for helpful discussions.

\section{References}

[1] X. Ren, H. Miao, Z. Peng, A review of cemented carbides for rock drilling: An old but still tough challenge in geo-engineering, Int. J. Refract. Met. Hard Mater. 39 (2013) 61-77.

[2] S. Norgren, J. Garcia, A. Blomqvist, L. Yin, Trends in the P/M hard metal industry, Int. J. Refract. Met. Hard Mater. 48(0) (2015) 31-45.

[3] S. Luyckx, A. Love, The dependence of the contiguity of WC on Co content and its independence from WC grain size in WC-Co alloys, , Int. J. Refract. Met. Hard Mater. 24(1-2) (2006) 75-79.

[4] M. Pellan, S. Lay, J.M. Missiaen, S. Norgren, J. Angseryd, E. Coronel, T. Persson, R. Bordia, Effect of Binder Composition on WC Grain Growth in Cemented Carbides, J. Am. Ceram. Soc. 98(11) (2015) 3596-3601.

[5] B. Roebuck, K.P. Mingard, H. Jones, E.G. Bennett, Aspects of the metrology of contiguity measurements in WC based hard materials, Int. J. Refract. Met. Hard Mater. 62 (2017) 161169.

[6] C.-S. Kim, G.S. Rohrer, Geometric and Crystallographic Characterization of WC Surfaces and Grain Boundaries in WC-Co Composites, Interface Sci. 12(1) (2004) 19-27.

[7] C.-S. Kim, T.R. Massa, G.S. Rohrer, Interface character distributions in WC-Co composites, J. Am. Ceram. Soc. 91(3) (2008) 996-1001. 
[8] M. Pellan, Development of grain boundaries and phase boundaries in WC-Co cemented carbides, Université Grenoble Alpes (France), 2015 (tel-01319087).

[9] M. Pellan, S. Lay, J.-M. Missiaen, S. Norgren, J. Angseryd, E. Coronel, T. Persson, EBSD

study to analyse mechanisms of phase boundary and grain boundary development in WC-Co cemented carbides, Powder Metal. 60(3) (2017) 208-215.

[10] S. Hagège, G. Nouet, P. Delavignette, Grain boundary analysis in TEM. IV. Coincidence and the associated defect structure in tungsten carbide, phys. stat. sol. (a) 62(1) (1980) 97107.

[11] M. Pellan, S. Lay, J.-M. Missiaen, S. Norgren, J. Angseryd, E. Coronel, T. Persson, A new insight into the $\Sigma=2$ grain boundary characteristics in WC powder and in WC-Co sintered materials, Acta Mater. 155 (2018) 372-378.

[12] M. Christensen, G. Wahnström, Co-phase penetration of WC(1 0 -1 0)/WC(1 0 -1 0$)$ grain boundaries from first principles, Phys. Rev. B 67(11) (2003) 115415.

[13] M. Christensen, G. Wahnström, Effects of cobalt intergranular segregation on interface energetics in WC-Co, Acta Mater. 52(8) (2004) 2199-2207.

[14] M.V.G. Petisme, S.A.E. Johansson, G. Wahnström, A computational study of interfaces in WC-Co cemented carbides, Modelling Simul. Mater. Sci. Eng. 23 (2015) 045001.

[15] J. Vicens, S. Lay, M. Benjdir, G. Nouet, Nature of the boundary planes in tungsten carbide-cobalt composites, J. Phys., Colloq. 51(C1, Intergranular Interphase Boundaries Mater.) (1990) 353-358.

[16] L. Akesson, An experimental and thermodynamic study of the Co-W-C system in the temperature range 1473-1698 K, University of Stockholm (Sweden), 1982.

[17] A. Delanoë, S. Lay, Evolution of the WC grain shape in WC-Co alloys during sintering: Cumulated effect of the $\mathrm{Cr}$ addition and of the $\mathrm{C}$ content, Int. J. Refract. Met. Hard Mater. 27(2) (2009) 189-197.

[18] F.J. Humphreys, Review Grain and subgrain characterisation by electron backscatter diffraction, J. Mater. Sci. 36(16) (2001) 3833-3854.

[19] A. Morawiec, Misorientation-angle distribution of randomly oriented symmetric objects, J. Appl. Cryst. 28(3) (1995) 289-293.

[20] O. Hardouin Duparc, J.-P. Couzinié, J. Thibault-Pénisson, S. Lartigue-Korinek, B.

Décamps, L. Priester, Atomic structures of symmetrical and asymmetrical facets in a near $\Sigma=9\{221\}$ tilt grain boundary in copper, Acta Mater. 55(5) (2007) 1791-1800.

[21] F. Sarrazit, F.R.C. Pond, N.A. Kiselev, Structure transition in a ZnO grain boundary, Phil. Mag. Lett. 77(4) (1998) 191-198.

[22] A.P. Sutton, V. Vitek, On the Structure of Tilt Grain Boundaries in Cubic Metals I.

Symmetrical Tilt Boundaries, Phil. Trans. Roy. Soc. of London. Series A, Mathematical and Physical Sciences 309(1506) (1983) 1-36.

[23] A.P. Sutton, V. Vitek, On the Structure of Tilt Grain Boundaries in Cubic Metals II. Asymmetrical Tilt Boundaries, Phil. Trans. Roy. Soc. of London. Series A, Mathematical and Physical Sciences 309(1506) (1983) 37-54.

[24] A.P. Sutton, V. Vitek, On the Structure of Tilt Grain Boundaries in Cubic Metals. III. Generalizations of the Structural Study and Implications for the Properties of Grain Boundaries, Phil. Trans. Roy. Soc. of London. Series A, Mathematical and Physical Sciences 309(1506) (1983) 55-68.

[25] D.L. Medlin, D. Cohen, R.C. Pond, Accommodation of coherency strain by interfacial disconnections at a $90^{\circ}<110>$ grain boundary in gold, Phil. Mag. Lett. 83(4) (2003) 223-232. 
[26] R.C. Pond, D.L. Medlin, A. Serra, A study of the accommodation of coherency strain by interfacial defects at a grain boundary in gold, Phil. Mag. 86(29-31) (2006) 4667-4684.

[27] W. Bollmann, Crystal lattices, interfaces, matrices: an extension of crystallography, Geneva, Bollmann, 1982.

[28] L. Priester, Grain boundaries and crystalline plasticity, Iste, Wiley, 2011.

[29] R.C. Pond, J.P. Hirth, Defects at Surfaces and Interfaces, in: H. Ehrenreich, D. Turnbull

(Eds.), Solid State Physics, Academic Press, 1994, pp. 287-365.

[30] R. Bonnet, M. Loubradou, HRPACK: a software describing the elastic fields near dislocations and interfaces at atomic scale, Ultramicroscopy 69(4) (1997) 241-257.

[31] R. Bonnet, Periodic displacement and stress fields near a phase boundary in the isotropic elasticity theory, Phil. Mag. A 43(5) (1981) 1165-1187.

[32] J.P. Hirth, J. Lothe, Theory of dislocations, McGraw-Hill, New York., 1968.

[33] W. Bollmann, Crystal defects and crystalline interfaces, Springer-Verlag, Berlin, 1970.

[34] A.H. King, D.A. Smith, The effects on grain-boundary processes of the steps in the boundary plane associated with the cores of grain-boundary dislocations, Acta Cryst. A 36(3) (1980) 335-343.

[35] J.P. Hirth, R.C. Pond, Steps, dislocations and disconnections as interface defects relating to structure and phase transformations, Acta Mater. 44(12) (1996) 4749-4763.

[36] J.P. Hirth, Dislocations, steps and disconnections at interfaces, J. Phys. Chem. Sol. 55(10) (1994) 985-989.

[37] R.C. Pond, D.A. Smith, On the absorption of dislocations by grain boundaries, Phil. Mag. A 36(2) (1977) 353-366.

[38] R. Bonnet, Periodic displacement and stress fields near a phase boundary in the isotropic elasticity theory, Phil. Mag. A 43(5) (1981) 1165-1187.

[39] G.H. Bishop, W.H. Hartt, G.A. Bruggeman, Grain boundary faceting of $<10-10>$ tilt boundaries in zinc, Acta Metall. 19(1) (1971) 37-47.

[40] C.B. Carter, $\Sigma=99$ and $\Sigma=41$ Grain boundaries, Acta Metall. 36(10) (1988) 2753-2760.

[41] M. Christensen, G. Wahnström, Strength and reinforcement of interfaces in cemented carbides, Int. J. Refract. Met. Hard Mater. 24(1-2) (2006) 80-88.

[42] S. Lay, M. Loubradou, Characteristics and origin of clusters in submicron WC-Co cermets, Phil. Mag. 83(23) (2003) 2669-2679.

Figure captions

Figure 1. Scanning electron microscopy images of (a) W2-10Co and (b) C2-50Co alloys sintered for $1 \mathrm{~h}$. The WC phase appears as bright grains surrounded by the Co rich binder in the back scattered electron mode.

Figure 2. Area fraction of [2-1 -1 0] boundaries as a function of Co content and sintering time for the two sets of alloys ( $C 2$ and W2). The area fraction was expressed per unit solid angle.

Figure 3. Rotation angle distribution for the [2 - 1 -1 0] axis (a) for the WC powder and (b) in the sintered alloys. The frequency in number is relative to the [2 -1 -1 0] grain boundaries. The plot of a random distribution in a hexagonal material is drawn as a dotted line [19]. 
Figure 4. Surface fraction of [ $2-1-10$ ] boundaries compared to all boundaries in the material with rotation angle equal to (a) $49^{\circ}$, (b) $60^{\circ}$ and (c) $90^{\circ}$. The tolerance angle on the rotation angle and axis direction is $5^{\circ}$.

Figure 5. (a) WC grain delimited by two basal facets (B) and three prismatic facets (P) in WCCo alloys. (b) Its projection along [ $2-1-10]$ with two $B$ and only one $P$ facet parallel to the [2

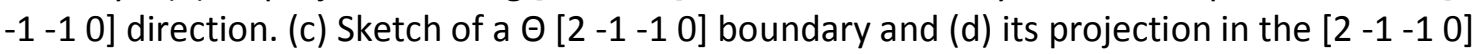
plane.

Figure 6: Examples of [ 2 -1 -10 ] grain boundaries viewed by TEM along the [2 - 1 -1 0 ] common direction in the W2-50Co alloy sintered for $5 \mathrm{~h}$. The boundary habit plane is a basal (a-b) or prismatic (c) facet parallel to a low-index plane.

Figure 7. (a) Projection of the WC crystal lattice along [2 -1 -1 0] showing the distribution of the $(0 \mathrm{~h}-\mathrm{h}$ I) planes. Large and small discs are $\mathrm{W}$ and $\mathrm{C}$ atoms, respectively, at height 0 or $1 / 6[21 ; 1 ; 0]$.

Figure 8. Examples of grain boundaries deviating from preferred misorientations in the W2$50 \mathrm{Co}$ alloy sintered for $5 \mathrm{~h}$. (a) Bright field image of a boundary with a rotation angle of $59.8^{\circ}$, associated diffraction patterns, and magnification of the habit plane. (b) Bright field image of the boundary with a rotation angle of $46.3^{\circ}$ and associated diffraction pattern. The magnification shows faceting of the habit plane.

Figure 9. HRTEM images of the $26.2^{\circ}$ [ 2 -1 - 10 ] grain boundary viewed along the [2 -1 - 10 ] direction in the WC-20Co, $\mathrm{Cr}, \mathrm{C}$ alloy. (a) A series of atomic planes in the crystal $\mu$ ending at the boundary is observed. (b) Similar atomic patterns are identified along the boundary.

Figure 10. Schematic diagram of the $29.41^{\circ}$ [ 2 - 1 - 1 0] grain boundary viewed along [2 -1 -1 0] direction. The mismatch between $\left[\begin{array}{llll}0 & 1 & -1 & 0\end{array}\right]_{\mu}$ and $\left[\begin{array}{llll}0 & 1 & -1 & -1\end{array}\right]_{\lambda}$ is equal to $13.8 \%$. Only atomic positions of $\mathrm{W}$ atoms (or equivalently $\mathrm{C}$ atoms) are represented on the drawing and empty

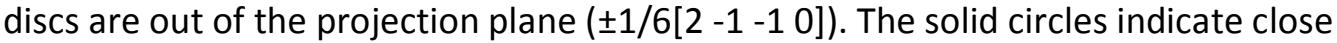
coincidence of the atom sites at the boundary and dotted circles a difference in height of $1 / 6[2-1-10]$. Basic lattice vectors ai ( $i=1$ to 3 ) and $c$ are drawn in the crystal $\mu$ ( $a 1=1 / 3[2-1$ $-10], a 2=1 / 3\left[\begin{array}{llll}-1 & 2 & -1 & 0\end{array}\right], a 3=1 / 3\left[\begin{array}{llll}-1 & -1 & 2 & 0\end{array}\right]$ and $\left.c=\left[\begin{array}{llll}0 & 0 & 0 & 1\end{array}\right]\right)$.

Figure 11. Magnification of the boundary in the BC region. (a) HRTEM image with a Burgers circuit drawn around points $B$ and $C$, (b) schematic diagram of the reference boundary with stretched $\mu$ crystal and lattice vectors expressed in Miller indices, (c) same Burgers circuit as in (a) drawn in the dichromatic pattern associated with the reference boundary.

Figure 12. Magnification of the boundary in the $B C$ region. (a) HRTEM image with two Burgers circuits drawn around IC and BI portions of the boundary, (b-c) same Burgers circuits drawn in the dichromatic pattern associated with the reference boundary.

Figure 13. Study of the boundary in the C-D region. (a) HRTEM image with two Burgers circuits drawn around the JD and CJ portions of the boundary, (b-c) same Burgers circuits drawn in the dichromatic pattern associated with the reference boundary. 
Figure 14. Study of the boundary in the A-B region. The elastic displacement field calculated for a periodic array of $-a 2_{\mu}$ (or $\left.a 3_{\mu}\right)+1 / 2 C_{\lambda}$ misfit dislocations is superimposed on the HRTEM image.

Figure 15: Dichromatic pattern associated with the reference boundary depicting step vectors attached to the $1 / 2 C_{\lambda}$ disconnection found in this work.

Figure 16. (a) Diagram describing the deviation of the experimental $26.2^{\circ}$ [ $2-1-10$ ] boundary from the reference boundary as defined in §3.3.1. (b) Dislocation spacing associated with $b_{1}$ and $b_{2}$ Burgers vectors as a function of the deviation angle. The dotted line corresponds to the observed boundary misorientation.

Figure 17. Projection of $\mathrm{W}$ lattices along the [2 -1 -1 0] direction for several observed boundary misorientations, where large and small disks correspond to height 0 and $1 / 2$, respectively. Red circles represent near coincident sites at the boundary. The values $d_{\lambda}$ and $d_{\mu}$ are used to calculate the misfit between the planes meeting at the boundary.

Table captions

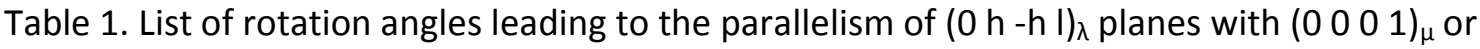
$\left(0 \begin{array}{lll}0 & -1 & 0\end{array}\right)_{\mu}$ planes.

Table 2. Determination of the Burgers vectors expressed in the $\lambda$ and $\mu$ crystals. Miller indices were used for intermediate calculations. 


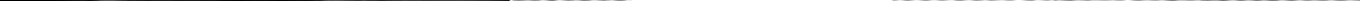

